\title{
Kommission Hüftsonografie nach Graf
}

Im Jahr 2012 wurden mehrere Dutzend Fähigkeitsausweise für weitere 5 Jahre rezertifiziert. In den letzten Monaten macht sich eine deutlich vermehrte Tendenz bemerkbar, dass sich in der Schweiz pädiatrisch tätige Kolleginnen und Kollegen aus dem nördlichen Nachbarland niederlassen. In der Regel beabsichtigen sie dann, auch die Hüftsonografien in der Schweiz durchzuführen. Die dazu notwendigen Vorbedingungen sind aber nur sehr unterschiedlich erfüllt. Dies bedingt auch einen erheblichen Aufwand für das Sekretariat, um alle erforderlichen Nachweise zu erhalten. Grundsätzlich muss jeder aus dem Ausland einreisende Hüftsonograf noch den schweizerischen Abschlusskurs absolvieren.

Erfreulich ist die Tatsache, dass am letzten Refresherkurs keine relevanten Beanstandungen mehr nötig waren, was doch ein Zeichen ist für die gestiegene Untersucherqualität.

Beat Dubs

Präsident Kommission Hüftsonografie SGUM/SSUM 